\title{
Assessing and comparing influencing factors of residual stresses in Selective Laser Melting using a novel analysis method Jean-Pierre Kruth ${ }^{1}$, Jan Deckers ${ }^{2, *}$, Evren Yasa ${ }^{3}$, Ruben Wauthlé ${ }^{4}$ ${ }^{1,2,3,4}$ Department of Mechanical Engineering, Catholic University of Leuven, Heverlee, Belgium
}

\section{$1 \quad$ Abstract}

2 During Selective Laser Melting, the irradiated material experiences large temperature 3 fluctuations in a short time which causes unwanted thermal stresses. In order to assess thermal stresses in a simple and fast way a new pragmatic method is developed, namely the Bridge

5 Curvature Method (BCM). The BCM is used to assess and qualitatively compare the influence of different laser scan patterns, laser parameter settings and more fundamental process changes on residual stresses. The results from the experiments as well as the findings from literature lead to two general conclusions: changes which reduce the high temperature gradient, like using short scan vectors and preheating of the base plate, reduce the thermal stresses. And, thermal stresses in a particular direction can be reduced by optimal choice of the orientation of scan vectors. The experiments indicate the reliability of the Bridge Curvature Method. Statistical analysis is used to check the repeatability of the method and to quantify the uncertainties during measurement.

\section{Keywords}

Additive manufacturing, Selective Laser Melting, residual stresses

Corresponding Author: Jan Deckers, Department of Mechanical Engineering, Catholic University of Leuven, Celestijnenlaan 300B, Box: 2420, B3001 Heverlee, Belgium, Email: jan.deckers@mech.kuleuven.be, Tel. +32 16 322772, Fax. +32 16322987 


\begin{tabular}{|c|c|c|}
\hline 1 & Notation & \\
\hline 2 & BCM & Bridge Curvature Method \\
\hline 3 & FDM & Fused Deposition Modelling \\
\hline 4 & LOM & Laminated Object Manufacturing \\
\hline 5 & REF. & reference part \\
\hline 6 & SLM & Selective Laser Melting \\
\hline 7 & $\mathrm{~T}$ & Thickness of bridge structure \\
\hline 8 & TGM & Temperature Gradient Mechanism \\
\hline 9 & W & Width of bridge structure \\
\hline 10 & $\alpha$ & angle of curvature $\left(^{\circ}\right)$ \\
\hline 11 & $\beta$ & angle between scan orientation and measurement datum $\left(^{\circ}\right)$ \\
\hline 12 & $\mathrm{Y}$ & angle between island orientation and measurement datum $\left({ }^{\circ}\right)$ \\
\hline 13 & $\varepsilon_{e l}$ & elastic strain (-) \\
\hline 14 & $\varepsilon_{p l}$ & plastic strain (-) \\
\hline 15 & $\varepsilon_{t h}$ & thermal strain $(-)$ \\
\hline 16 & $\sigma_{c o m p}$ & compressive stress $(\mathrm{Pa})$ \\
\hline 17 & $\sigma_{\text {res }}$ & residual stress $(\mathrm{Pa})$ \\
\hline 18 & $\sigma_{t e n s}$ & tensile stress $(\mathrm{Pa})$ \\
\hline 19 & $\sigma_{\text {yield }}$ & yield stress $(\mathrm{Pa})$ \\
\hline
\end{tabular}




\section{Introduction}

2 Selective Laser Melting (SLM) is a layered manufacturing process that allows building complex parts, lightweight and customized parts by consolidating successive layers of powder via melting with a laser. Since during the SLM process the powder material is fully melted, a low degree of porosity and good mechanical properties can be achieved.

6 During the SLM process, the material experiences large localised temperature fluctuations in a short time. This causes high thermal and resulting residual stresses which can introduce part warpage, cracks or an undesired reduction of strength. The goal of the reported investigation is to reduce the thermal stresses by changing the scan pattern, and altering process parameters like the layer thickness, pre-scanning a deposited powder layer or post-scanning an already scanned layer. To help achieve this goal, a new method is defined which enables assessing thermal stresses in a fast way by measuring the curvature of test parts when releasing them from the base plate on which they are built. The method enables assessing and comparing thermal stresses qualitatively. Thermal stresses are stresses induced in a body when some or all of its volume is not free to expand or contract in response to changes in temperature. A material in its liquid phase doesn't contain thermal stresses. In the SLM process, thermal stresses arise in solidified material, subjected to temperature gradients, or subjected to shrinkage caused by the solidification of adjacent laser molten material.

Residual stresses arise by non-uniform plastic deformations in solid material. As long as thermal stresses are elastic, no residual stresses will remain when the temperature comes back to its original value. If the thermal stresses inside a material become higher than the yield stress, residual stresses will remain after cooling. The sign $(+/-)$ of the remaining residual stress will always be the opposite of the sign of the thermal stress. As a decrease of thermal stress will 
reduce the residual stresses, the terms 'thermal stress' and 'residual stress' are somewhat coupled. In the remaining of the paper, these terms will be used interchangeably.

\subsection{Mechanisms of thermal stresses in SLM}

4

5

During the SLM process, the laser beam heats and melts material and causes a temperature gradient in the solid material being irradiated, resulting in residual stresses after cool-down.

Residual stresses in SLM are often larger than in e.g. Selective Laser Sintering (SLS) because SLM parts are built onto a thick and stiff base plate in order to avoid large part distortions when a successive layer shrink on top of the previous one. As seen in Fig. 1, the part produced will crack if the residual stresses are too high.

Mercelis [1] uses two descriptive models to explain the mechanism of the thermal stresses in SLM: the Temperature Gradient Mechanism (TGM) model and the cool-down phase model.

The TGM model states that the laser beam heats up solid material being irradiated during the SLM process, which as a result tends to expand as shown in Fig. 2 (a). The thermal expansion $\left(\varepsilon_{t h}\right)$ is partially inhibited by the surrounding colder material, yielding a compressive stress/strain condition in the irradiated zone. If the compressive stress exceeds the compressive yield stress ( $\left.\sigma_{\text {yield }}\right)$ of the material, the compressive strain will be partially elastic $\left(\varepsilon_{e l}\right)$ and partially plastic $\left(\varepsilon_{p l}\right)$ as illustrated in Fig. 2 (b).

After the laser beam leaves that area, the irradiated zone will cool and tend to shrink. The shrinkage is partially inhibited as a consequence of the plastic deformation developed during heating, yielding a residual tensile stress $\left(\sigma_{\text {tens }}\right)$ condition at the irradiated zone. According to the equilibriums of force and momentum of the part, the irradiated zone will become surrounded by a zone of compressive stress $\left(\sigma_{\text {comp }}\right)$ (Fig. 3).

The cool-down phase model describes the formation of residual stress as it arises in previously molten material when it re-solidifies and shrinks in solid state. The shrinkage is partially inhibited by the underlying material, thus introducing tensile stresses in the added top layer. 


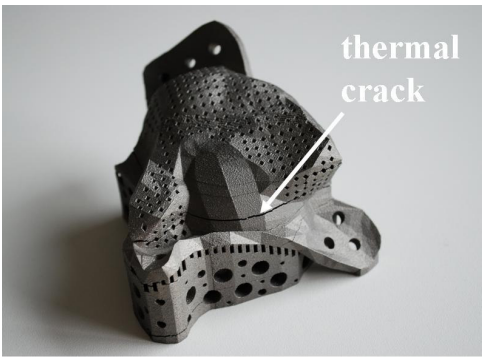

2 Fig. 1. Selective Laser Melted part with cracks induced by thermal stresses.

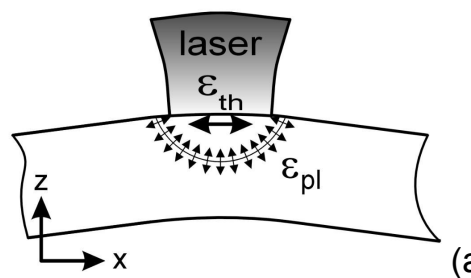

(a)

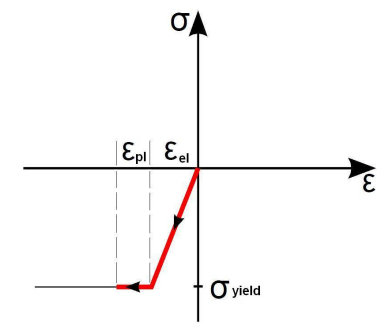

(b)

Fig. 2. (a) Induced stresses and deformation (strain) during laser beam heating [1] (b) Simplified 5 representation of the formation of thermal stress and strains in the irradiated zone.

6

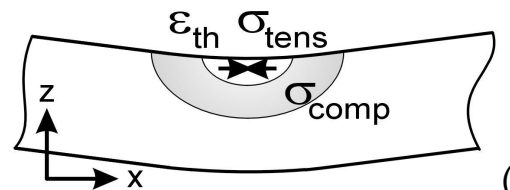

(a)

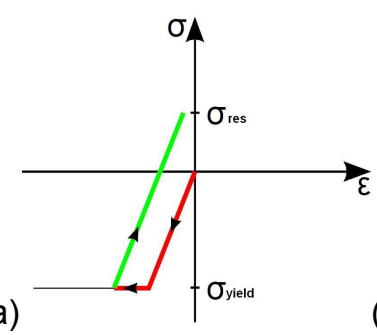

(b)

$7 \quad$ Fig. 3. (a) Occuring stresses and deformation (strain) when the part cools down [1]. (b)

8 Simplified representation of the formation of residual stresses and strain in the irradiated zone.

9

\subsection{Modelling of thermal stresses in SLM}

Mercelis [1] and Shiomi et al. [2] have defined a similar simplified mechanical model which quantifies the descriptive cool-down phase model by using the equilibriums of force and momentum. They assume the residual stresses in the latest added layer equal to the yield strength of the material. This causes high tensile stresses in the SLM part, compressive stresses in the upper part of the base plate and lower tensile stresses in the lower part of the 
base plate as illustrated in Fig. 4. The high tensile stresses inside the SLM part partially relax when removing the part from the base plate. The occurring relaxation consists of a constant term, which effects a uniform shrinkage of the part produced, and a linear term, which effects the bending of the produced part. The stresses in the part, which remain after relaxation, are considered as residual stresses (Fig. 5).

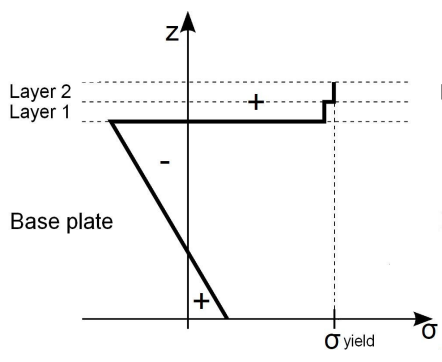

(a)

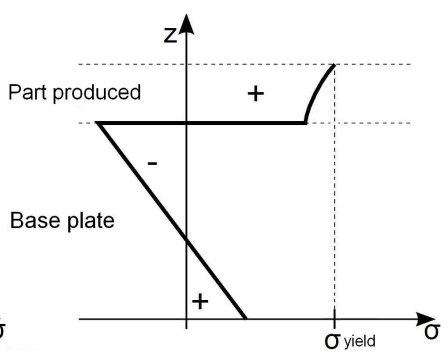

(b)

Fig. 4. (a) Residual stresses after adding two layers of molten powder on a base plate. (b) Residual stresses of an SLM part on a base plate after melting more or all layers forming the part. (simplified model)

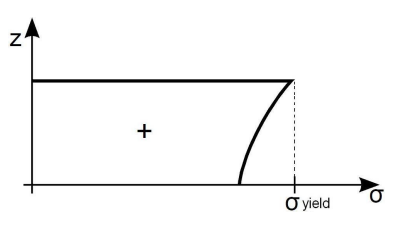

(a)

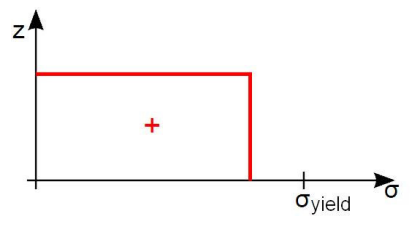

(b)

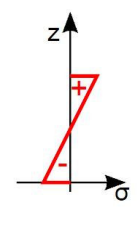

(c)

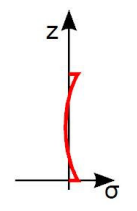

Fig. 5. (a) Residual stresses within an SLM part still connected to the base plate. (b) Constant and linear relaxation terms. (c) Residual stresses in an SLM part removed from the base plate, i.e. (a)-(b).

Mechanical models simplify the heat input of the laser. In order to have more accurate models, it is necessary to calculate the exact temperature distribution in the part during processing. Many authors have discussed thermal modelling of other laser processing techniques like laser welding [3] and laser forming [4]. Thermal modelling of the SLM process is similar, but the 
powder characteristics and the scanning pattern of the laser beam also have to be taken into account.

Since residual stresses do not only depend on the temperature history, thermal models cannot predict the resulting residual stresses without mechanical considerations. Thermomechanical models are able to calculate a resulting stress profile from a thermal model. Many authors use the ABAQUS finite element package for creating thermomechanical models. Nickel et al. [5] used the finite element package to examine the effect of deposition patterns on the resulting stresses and deflections in laser deposited metal parts. Li et al. [6] used the package to investigate thermal stresses and their implication on cracking during laser melting of ceramic materials. Some researchers use their own written methods (e.g. Osakada and Shiomi, [7]) or use another packages like MARC MENTAT (Over et al., [8]) or SYSWORLD (Niebling and Otto, [9]).

As illustrated by Bhadeshia [10], many relations between material properties and thermal stresses are well understood. However, all this knowledge has not been extensively implemented yet for the calculation of thermal stresses.

\subsection{Measuring residual stresses}

Besides modelling the SLM process, many researchers have performed experimental tests to measure the residual stresses. These tests aim to verify the results of the theoretical models, or to examine the influence of non-simulated phenomena.

As illustrated by Withers and Bhadeshia [11] and by Kandil et al. [12], many techniques for measuring the residual stresses in a part are available. Usually a classification is made in two groups, whether the measurement is destructive or not. Destructive methods include the layer removal method, the crack compliance method [1], the contour method [13] and the hole drilling method. Non-destructive methods include X-ray diffraction, neutron diffraction, ultrasonic and magnetic measuring methods. 


\subsection{Purpose of current work}

Modeling thermal stresses and detailed investigation of thermal stresses by experiments can be time consuming. On the other hand, qualitative information of thermal stresses may be sufficient in SLM for comparing stress magnitude and for selecting build strategy and parameters minimizing stress levels. The purpose of this paper is to introduce a new pragmatic method (Bridge Curvature Method, BCM) for simple, fast and still accurately analysing possible strategies to reduce residual stresses during SLM. The Bridge Curvature Method resembles the layer removal methods (see §1.3). Bridge-like structures are produced on a base plate using SLM. When removing the bridge structure from the base plate, the amount of resulting curvature is used as a measure for the amount of residual stresses in the part after production.

In a first set of experiments, statistical analysis is used to optimise the geometry of the bridges for repeatable measurement results. The reliability of this geometry and of the Bridge Curvature Method in general is proven in two ways. Firstly, the curvature of reference bridges are measured in order to investigate the repeatability. Secondly, the results, obtained by investigating the influence of different stress reducing strategies with Bridge Curvature Method, are compared with results from literature.

The Bridge Curvature Method enables qualitative comparisons possible stress reducing strategies in a fast and easy way. For example the Bridge Curvature Method can be used to identify qualitatively the benefit of a certain island scanning strategy with a particular stress relieving furnace treatment, or to compare two stress relieving furnace treatments with different parameters, etc.

\section{Description of the novel analysis method and equipment}

The first section below describes the main equipment used in this paper. The second section describes the Bridge Curvature Method and illustrates the influence of bridge geometry on the repeatability of the method. 


\begin{tabular}{|c|c|c|}
\hline Machine & KUL-SLM machine & Concept Laser M3 Linear Machine \\
\hline Laser type & Yb:YAG & $\mathrm{Nd}: Y A G$ \\
\hline Laser wavelength & $1085 \mathrm{~nm}$ & $1064 \mathrm{~nm}$ \\
\hline Spot size $(99 \%)$ & $80 \mu \mathrm{m}$ & $180 \mu \mathrm{m}$ \\
\hline Maximum power & $300 \mathrm{~W}$ & $100 \mathrm{~W}$ \\
\hline Build envelope & $\varnothing 100 \mathrm{~mm} \times 100 \mathrm{~mm}$ & $250 \mathrm{~mm} \times 250 \mathrm{~mm} \times 250 \mathrm{~mm}$ \\
\hline
\end{tabular}

Besides the equipment to produce the parts, a non-contact vision measuring system (Mitutoyo

Since the easiness of implementing stress reducing strategies is machine dependent, two different machines are selected to produce the test parts: an in-house developed SLM machine and a Concept Laser M3 Linear machine. The benefit of the in-house developed SLM machine is that its software enables more convenient control over the scan vectors to investigate new scan strategies (e.g. using short scan vectors). On the other hand some scanning algorithms (e.g. island scanning) are not implemented in the in-house machine. For these algorithms the Concept Laser M3 Linear machine is used.

In order to investigate the reliability of the proposed method, not only two different machines are used, but also two different materials. To avoid powder contamination, one type of powder is used in each machine. Ti-6Al-4V test parts are produced using an in-house developed SLM machine and AISI 316 L stainless steel test parts are produced using a Concept Laser M3 Linear machine. Table 1 gives an overview of the properties of these machines.

Table 1.

Properties of the KUL-SLM machine and the Concept Laser M3 Linear Machine.

\subsection{Equipment}




\subsection{Bridge Curvature Method (BCM)}

As illustrated in Fig. 6 (a), for using the Bridge Curvature Method, a bridge like part has to be built on a base plate. When the test part is cut off the base plate by e.g. wire EDM (electrical discharge machining), the bridge curls because of the relaxation of internal stresses [1]. Fig. 6

(a) and Fig. 6 (b) illustrate the method. The planes at the bottom of the pillars of the bridge deviate from their normal position and form a curling angle a which is indicative of the thermal stresses. The lines which determine the curling angle $\alpha$ and the curling angle $\alpha$ itself are automatically determined by the measurement software of the Mitutoyo Quick Vision Pro 202 system. For each test part, the curling angle is fastly measured 10 times (5 times on each side of the part). From the measured data the mean value and standard deviation are calculated together with a $95 \%$ confidence interval. Notice that only the residual tensile stresses in the $x$ direction at the top middle of the part (i.e. at the bridge overhang) cause the bending and that the Quick Vision measurements can be executed very fast (only few minutes). In a first set of experiments, 4 bridge structures with different geometries (width 'W' and thickness 'T', see Fig. 6 (c) and Table 2) were each produced 2 times on the KUL-SLM machine with standard SLM parameters, optimized for density (see Table 3). After measuring the curling angle of each produced bridge structure for 10 times as described above, a Fisher tests and Student tests are used to determine which geometry gives the most repeatable measurement results. As a result both bridge 1 and bridge 2 had geometries which could be used further to investigate the residual stresses. Bridge geometry 2 is finally chosen to become the reference geometry, because it can be produced faster compared to geometry 1 due to a smaller build height (smaller ' $T$ ' value).

Since bridge 2 is produced with parameters optimized for density and geometry, it is called the 'reference part'. All other bridge structures in this paper are produced with the geometry of the reference part (see Fig. 6 (d)). 
2
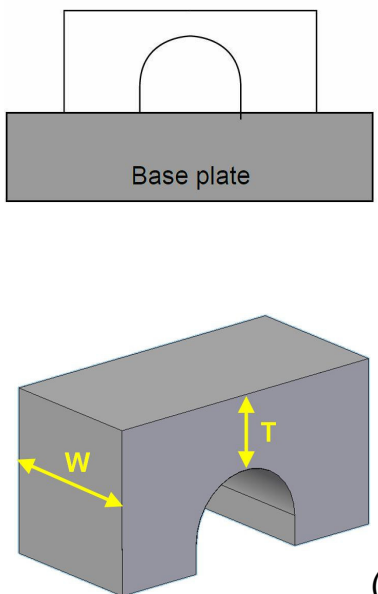

(c)

(a)
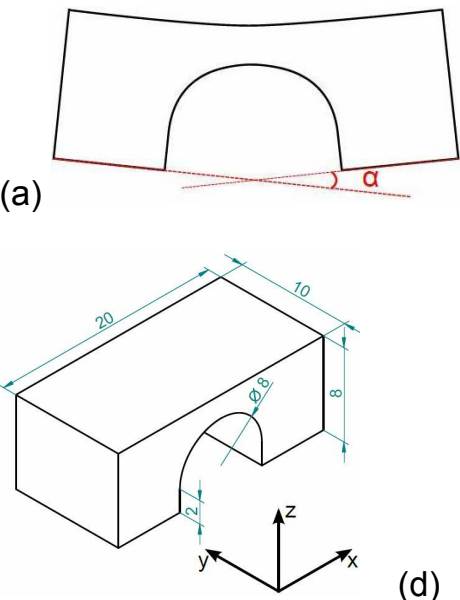

(b)

(d)

4 Fig. 6. Principle of the method for identifying the residual stresses in the test parts. (a) Before and (b) after removal from base plate. Geometry of the test parts: (c) Variable width 'W' and thickness ' $T$ ' to optimise bridge structure for repeatability. (d) Final dimensions of optimised

7 bridge geometry $[\mathrm{mm}]$.

$8 \quad$ Table 2.

9 Different width 'W' and thickness ' $T$ ' of bridge structures in a geometrical study.

\begin{tabular}{ccc}
\hline & $\mathrm{W}[\mathrm{mm}]$ & $\mathrm{T}[\mathrm{mm}]$ \\
Bridge 1 & 10 & 5 \\
Bridge 2 & 10 & 2 \\
Bridge 3 & 4 & 5 \\
Bridge 4 & 4 & 2 \\
\hline
\end{tabular}

During each build the reference part is made in order to investigate the repeatability of the Bridge Curvature Method. In all graphical representations of the curling angle $\alpha$, the curling angle of the reference part is illustrated and indicated with 'REF.'. Table 3 and Table 4 show the obtained mean values and standard deviations (95\% confidence interval, Student test) for the curling angle $\alpha$, considering all produced reference parts. A more detailed uncertainty analysis of the method (e.g. the influence of a bad EDM cut on the measured angle) is described by

17 Wauthlé [14]. 
Table 3.

2 Reference parameters used to build Ti-6AI-4V test parts on the KUL-SLM machine.

\begin{tabular}{lc}
\hline Material & Ti-6Al-4V \\
Scan vectors & x-direction, bi-directional \\
Hatch spacing & $74 \mu \mathrm{m}$ \\
Scan speed & $225 \mathrm{~mm} / \mathrm{s}$ \\
Laser power & $42 \mathrm{~W}$ \\
Contour scan & before fill \\
Layer thickness & $30 \mu \mathrm{m}$ \\
Reference value & $\alpha_{\text {ref, LM }}=2,797^{\circ} \pm 0,033^{\circ}$ \\
\hline
\end{tabular}

3 Table 4.

4 Reference parameters used to build AISI 316L stainless steel test parts on the Concept Laser 5 machine.

\begin{tabular}{lc}
\hline Material & 316L stainless steel \\
Scan vectors & x-direction, bi-directional \\
Hatch spacing & $126 \mu \mathrm{m}$ \\
Scan speed & $380 \mathrm{~mm} / \mathrm{s}$ \\
Laser power & $100 \mathrm{~W}$ \\
Contour scan & before fill \\
Layer thickness & $30 \mu \mathrm{m}$ \\
Reference value & $\alpha_{\text {ref, LM }}=1,333^{\circ} \pm 0,024^{\circ}$ \\
\hline
\end{tabular}

\section{EXPERIMENTS AND RESULTS}

7 The first section describes the influence of the laser scan pattern on the measured curling angle

8 of the test parts. Laser scan strategies can be adapted in a relatively easy way by changes in the

9 scanning software. The second section describes the influence of more fundamental process

10 changes which are not yet commonly used for SLM.

\section{$11 \quad 3.1$ Change in laser scan pattern}

12 Length of the scan vectors

13 The parallel bi-directional scan vectors, often used in SLM, do not always have the same length.

14 The first test checks the influence of the vector length $(I)$ in the $x$-direction on the bending of the part and is performed on the KUL-SLM machine. Fig. 7 shows the scan pattern used to investigate the influence of shorter scan vectors on the thermal stresses. The sequence of the vectors scanned by the laser is determined by the path of the dotted line. 
1 The results for vector lengths are shown in Fig. 8. Three test parts with shorter vector lengths 2 are compared to the reference part which has a vector length of $20 \mathrm{~mm}$ (indicated by REF.). 3 There is almost no difference in the measured angle between vectors of $20 \mathrm{~mm}$ and $10 \mathrm{~mm}$. At 4 lower vector lengths, the measured angle decreases. This effect is more pronounced if the vector length is reduced further. Vector lengths of $2 \mathrm{~mm}$ record the largest improvement of $13 \%$ reduction of curling angle $\alpha$, as compared with the curling angle of the reference part.
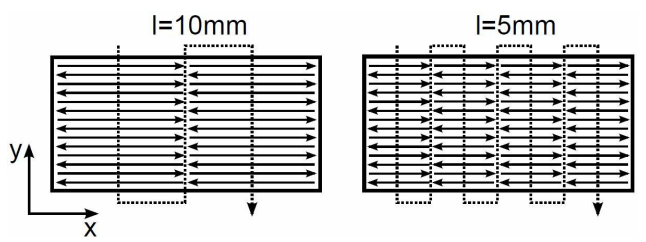

$8 \quad$ Fig. 7. Scan pattern to investigate short scan vectors.

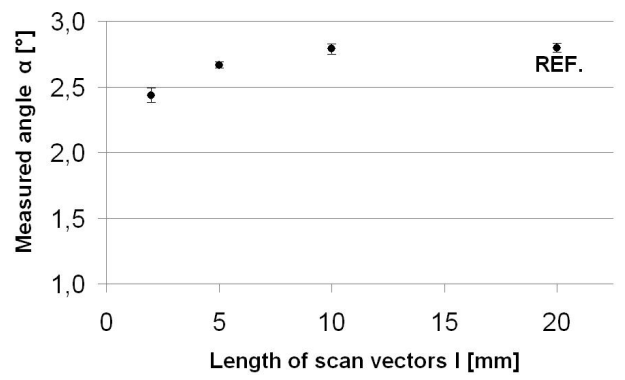

Fig. 8. Influence of vector length (I) in the $\mathrm{x}$-direction on curling of Ti-6Al-4V test parts $(\mathrm{REF} .=$ reference part).

\section{Orientation of the scan vectors}

To see the influence of the orientation of the parallel scan vectors on the measured angle $\alpha$, different test parts are made on the KUL-SLM machine, in which the angle $\beta$ between the parallel scan vectors and the measurement datum (x-direction) changes from $0^{\circ}$ (reference) to $90^{\circ}$. Fig. 9 illustrates this rotation for $45^{\circ}$ and $90^{\circ}$.

The measured curling angle $\alpha$ decreases as the rotational angle is changed from $0^{\circ}$ to $90^{\circ}$ (Fig. 10). The bending of the test part reduces by $59 \%$ if the scan vectors are oriented $90^{\circ}$ from the 
curling measurement datum. If the parallel scan vectors are layer-wise alternated $\left(\beta=0^{\circ}-90^{\circ}\right)$,

2 the reduction of the measured angle is found to be $45 \%$.

3 Notice that the length of the vectors also changes during this rotation of the scan vectors. Due to 4 the small difference in curling angle for test parts with vector lengths of $20 \mathrm{~mm}$ and $10 \mathrm{~mm}$ (Fig.

5 8), this change in vector length (I) has a negligible influence on the obtained results.

6

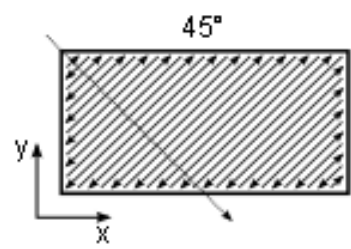

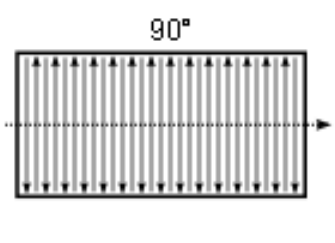

$7 \quad$ Fig. 9. Scan pattern to investigate the orientation $\beta$ of the scan vectors.

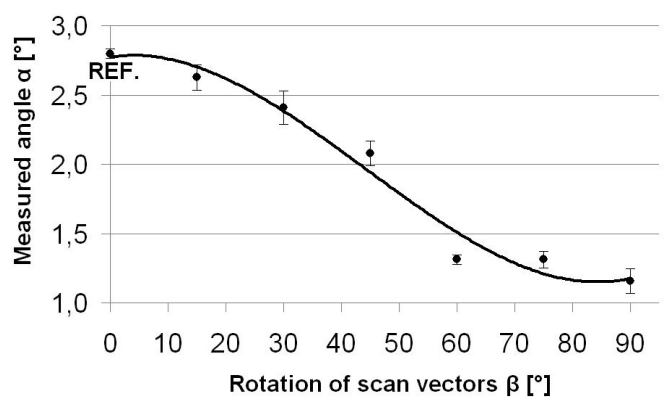

8

9 Fig. 10. Influence of the orientation $\beta$ of the scan vectors on the curling of the test part.

Island scanning

Island scanning is a scan strategy patented by Concept Laser $\mathrm{GmbH}$. This scan strategy divides the area to be scanned into small square islands. The sequence in which the islands are scanned is chosen randomly. The special slicing algorithm embedded in Magics $®$ software allows to change the size of the islands, the orientation of the islands, and it is also possible to shift the islands in the $\mathrm{x}$ - and $\mathrm{y}$-direction between different layers. Fig. 11 shows a possible scan pattern for islands of $5 \times 5 \mathrm{~mm}$ and a $15^{\circ}$ rotation $\mathrm{y}$ from the $\mathrm{x}$-direction. 


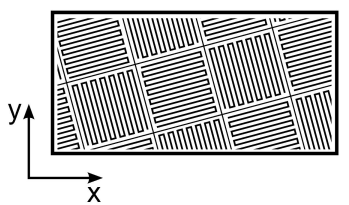

4 Fig. 11. Possible scan pattern for island scanning with islands of $5 \times 5 \mathrm{~mm}$ and a $15^{\circ}$ rotation $\mathrm{y}$.

5 The effect of the island size is shown in Fig. 12 for islands rotated $15^{\circ}$ from the x-direction. The

6 use of island scanning reduces the measured angle $\alpha$, but the size of the islands doesn't seem

7 to influence the results. Fig. 13 illustrates the effect of the rotation $y$ for islands of $5 \times 5 \mathrm{~mm}$. If the

8 rotation $\mathrm{y}$ is $45^{\circ}$ instead of $15^{\circ}$, the measured angle further decreases. Island scanning with

9 islands of $5 \times 5 \mathrm{~mm}$, rotated $45^{\circ}$ from the $\mathrm{x}$-direction reduces the measured angle by $36 \%$.

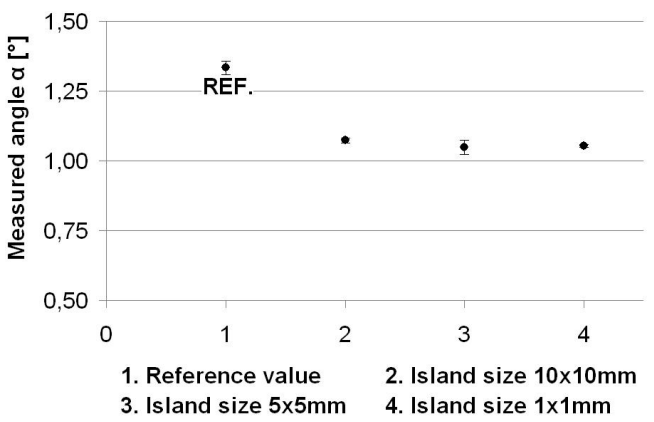

11 Fig. 12. Results of island scanning with different island size on the Concept Laser machine.

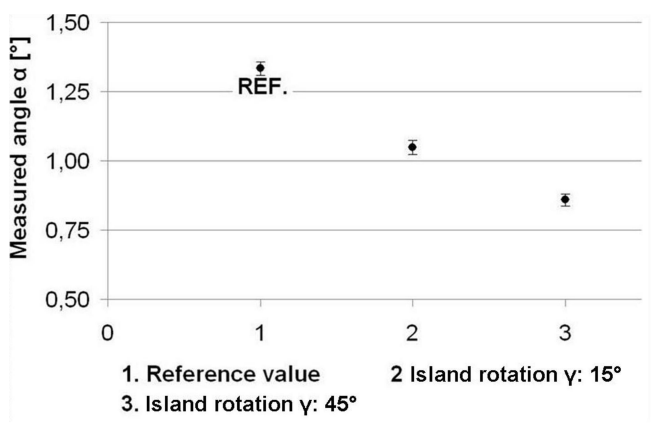

13 Fig. 13. Results of island scanning with different island orientations $y$ on the Concept Laser machine. 


\subsection{Process changes}

\section{Post-scanning}

Post-scanning means re-scanning a molten and consolidated layer: each layer is first melted using the reference parameters given in Table 3 . Then the layer is re-scanned with the same scan pattern and spot size. The results for post-scanning of Ti-6Al-4V parts with different scan speeds on the KUL-SLM machine are shown in Fig. 14. In this figure, the value at $0 \mathrm{~mm} / \mathrm{s}$ is the reference value without post-scanning (SLM scan speed of $225 \mathrm{~mm} / \mathrm{s}$ ).

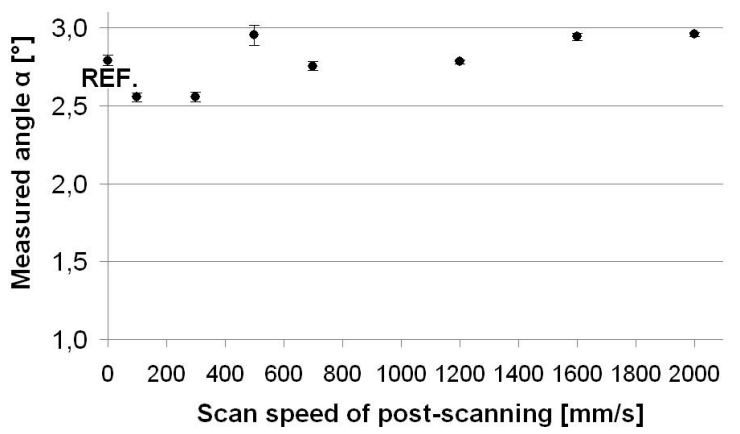

Fig. 14. Results of post-scanning with different scan speeds on the KUL-SLM machine.

Only the low scan speeds, involving remelting, reduce the measured angle slightly. A machine using post-scanning speed of $100 \mathrm{~mm} / \mathrm{s}$ gives the maximum reduction of $8 \%$. Also some $316 \mathrm{~L}$ test parts were produced on the Concept Laser M3 Linear machine, but none of these parts showed any noticeable reduction in curling angle [14].

Pre-scanning

When pre-scanning is applied, the laser first sinters the powder material, before melting the material completely with the reference parameters of Table 3 (Ti-6Al-4V and $225 \mathrm{~mm} / \mathrm{s}$ ). The results for pre-scanning with $800 \mathrm{~mm} / \mathrm{s}$ and $1600 \mathrm{~mm} / \mathrm{s}$ speeds and a constant laser power of 42 W on the KUL-SLM machine are presented in Fig. 15 . The value at $0 \mathrm{~mm} / \mathrm{s}$ is the reference value without pre-scanning. Pre-scanning reduces the measured angle $\alpha$ only slightly with a maximum reduction of $6 \%$ obtained at $800 \mathrm{~mm} / \mathrm{s}$. 


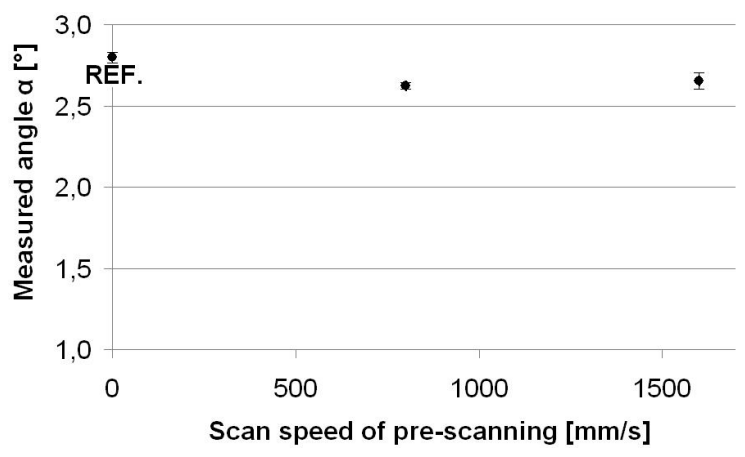

2 Fig. 15. Results of pre-scanning with different scan speeds on the KUL-SLM machine.

3 Different layer thickness

4 Larger layer thicknesses can be used to increase the production rate of the process. However,

5 the improvement in the productivity is limited as the layer thickness cannot exceed the melting

6 depth. If the powder layer is too thick, the laser beam will not be able to fully penetrate and melt

7 it, inducing unwanted porosities which affect the mechanical properties in an undesired way.

8 Other process parameters (e.g. scan speed and scan spacing) are influential on optimizing the

9 layer thickness for productivity. Since the stress normal to a pore is zero, porosities tend to decrease the residual stresses.

The scan speed needed to produce optimal density parts was not known for layer thicknesses other than $30 \mu \mathrm{m}$. Therefore multiple test parts with a layer thickness of $60 \mu \mathrm{m}$ but with different scan speeds (v) were produced on the Concept Laser machine. For each part produced, the curling angle is measured (Fig. 16). Also the part densities are measured with the Archimedes weighting method (Table 5) and the cross-sections are investigated by an optical microscope (Fig. 17).

A low scan speed is expected to heat up more material around the laser spot and to reduce in this way the temperature gradient and related thermal stresses. In contrary to this expectation, Fig. 16 shows only a decrease in curling angle for the higher scan speeds of $250 \mathrm{~mm} / \mathrm{s}$ and 380 $\mathrm{mm} / \mathrm{s}$. Since part $\mathrm{n}^{\circ} 3(250 \mathrm{~mm} / \mathrm{s})$ has a rather high density value, the lower curling angle for its 
scan speed cannot be due to stress relaxation caused by a large amount of pores in the part. It

2 seems to be difficult to compare the influence of layer thickness on thermal stresses without

3 taking into account the combined influence of part density and scan parameters (in this case the scan speed) to achieve this density. On the other hand, it can be stated that the measured angle of the test part with a density closest to the density of the reference part (part $n^{\circ} 3$ ) and a layer

6 thickness of $60 \mu \mathrm{m}$ gives a reduction of $6 \%$.

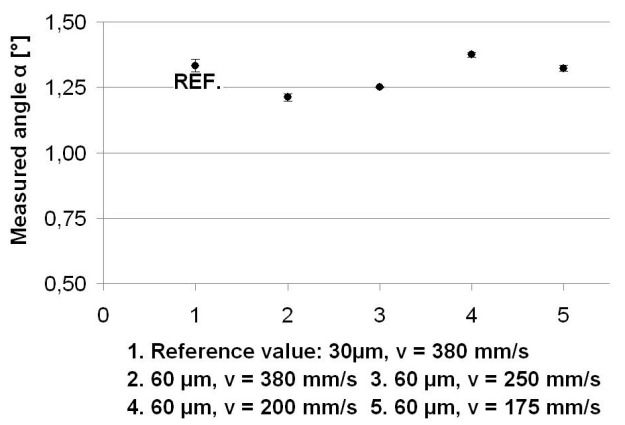

8 Fig. 16. Influence of layer thickness and scan speed on the curling of the test part.

$9 \quad$ Table 5.

10 Density of the test parts produced with a layer thickness of $60 \mu \mathrm{m}$ and different scan speeds.

\begin{tabular}{lccc}
\hline & scan speed [mm/s] & density [\%] & angle $\bar{\alpha}\left[^{\circ}\right]$ \\
1 (ref.) & 380 & 97,57 & 1,333 \\
2 & 380 & 95,83 & 1,212 \\
3 & 250 & 98,23 & 1,251 \\
4 & 200 & 98,82 & 1,374 \\
5 & 175 & 98,88 & 1,323 \\
\hline
\end{tabular}
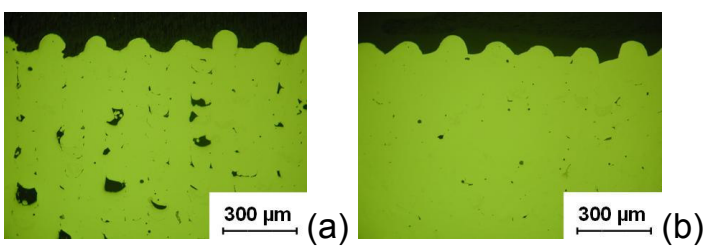

12 Fig. 17. Optical microscope images of cross-sections of $60 \mu \mathrm{m}$ layer thickness parts. (a) $\mathrm{v}=380 \mathrm{~mm} / \mathrm{s},(\mathrm{b}) \mathrm{v}=175 \mathrm{~mm} / \mathrm{s}$. 
Preheating of the base plate

In order to investigate the influence of preheating on residual stresses, the base plate is heated to $180^{\circ} \mathrm{C}$. This resulted in a reduction of $10 \%$ of the measured angle of curvature of the SLM part.

\section{Heat treatment}

Heat treatments relieve the residual stresses developed during fabrication [15]. Fig. 18 shows the temperature cycle that is applied to a Ti-6Al-4V reference part on the baseplate, produced on the KUL-SLM machine (Table 3). This heat treatment reduces the measured angle by $80 \%$.

Notice that heat treatments are post-processing techniques allowing to reduce thermal stresses, but cannot be used to avoid thermal cracking during the building of a part.

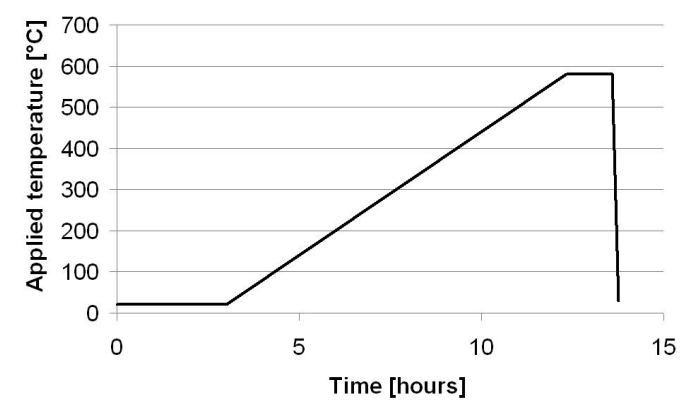

Fig. 18. Temperature cycle for heat treatment.

\section{DISCUSSION}

The Bridge Curvature Method (BCM) is developed and used as a novel analysis method to investigate the thermal stresses formed during SLM. The method can be used to assess and qualitatively compare thermal stresses in other layered manufacturing processes which use a base plate, like Fused Deposition Modelling (FDM), Laminated Object Manufacturing (LOM), etc.

8 The experiments revealed that scanning with short scan vectors reduces the thermal stresses.

9 Tests with Ti-6Al-4V demonstrated that if the length of the scan vectors is $2 \mathrm{~mm}$ instead of 20 $20 \mathrm{~mm}$, the measured angle of curvature produced after cutting the part from the base plate is 21 reduced by $13 \%$. Increasing the process temperature by preheating the base plate up to $180^{\circ} \mathrm{C}$ 
reduces the curling angle by $10 \%$. Both results indicate that changes which lower the thermal gradients reduce the thermal stresses: shorter scan vectors experience a smaller temperature gradient due to the higher remnant heat of the previous scan line. Preheating the base plate also reduces the temperature gradient during scanning. These findings are in agreement with the temperature gradient mechanism (TGM) described in literature (see §1.1), which indicates the reliability of the Bridge Curvature Method. The tests in §3.1 'Orientation of the scan vectors' and 'Island scanning' show that the magnitude of the thermal stresses differs as the angle $\beta$ between the scan vectors and the curvature measurement datum ( $\mathrm{x}$-direction) changes. Thermal stresses in the direction perpendicular to the scan vectors are lower than thermal stresses parallel to the scan vectors. Thermal stresses in a particular direction can be reduced by optimal choice of the orientation of scan vectors. Nickel et al. [5] observes similar results by making a thermomechanical model and doing similar experiments to investigate the relation between thermal stresses and deposition patterns in rapid manufacturing. Again the reliability of the Bridge Curvature Method is indicated by comparing the obtained results with results from literature.

Notice that using the effect of scan orientation simply moves the problem to another axis. In order to avoid thermal deformation, one has to orientate the scan vectors always to the direction with the highest resistance against that deformation (i.e. highest stiffness). On the other hand one has to keep in mind that if thermal stresses cannot be tempered during production, they can induce cracks before the part is completed.

Post-scanning and pre-scanning with the same laser beam spot size and the same scan pattern could only reduce the measured angle by respectively $8 \%$ and $6 \%$. These reductions are considered to be relatively low because the same mechanism which induces the high thermal stresses during the melting step will induce thermal stresses during the post-scanning and prescanning step. 
1 The influence of the stress reducing strategies on the mechanical properties is only partially 2 studied. For example, Yasa [16] demonstrated the effect of post-scanning with 316L stainless 3 steel on toughness by using Charpy impact tests. The results show that applying post-scanning 4 (i.e. re-melting of every SLM layer with the parameters utilized during SLM) improves toughness 5 because it reduces part porosity. During SLM of metals, the density of the parts is the most 6 important metallurgical parameter which determines the mechanical properties. The SLM 7 parameters used to produce the reference parts (see Table 3 and Table 4) are optimized for density. It is observed that during the tests performed in this paper (except the tests with different layer thickness, see §3.2) the density of the parts produced is not less than the densities of the reference parts. The influence of stress reducing strategies (e.g. furnace treatment) on other metallurgical parameters (e.g. undesirable carbide formation) is still open for future research.

Other than optimising the bridge geometry (i.e. an overhang structure) for repeatability, the influence of geometry on thermal stresses during SLM is not thoroughly investigated in the scope of this study. Other geometries may be employed to assess the residual stresses qualitatively. Alternatively, thermomechanical models can be used if validated correctly.

The proposed method gives only information about thermal stresses after the production of a part while it is still connected on the base plate. No direct information about what happens during production is given. The stress reducing strategies investigated in this paper are strictly limited to the case where the part doesn't fail during production. Thermal, mechanical or thermomechanical models can be used to correlate thermal stresses during and after the production of a part. For example, the TGM model (see §1.1) states that high stresses during laser irradiation lead to more plastic deformation, and more plastic deformation during production leads to higher thermal stresses after production. The TGM model states that stresses during and after production have opposite signs and that their absolute values are more 
or less proportional. Other models (see §1.2) can be used to investigate the influence of other effects, e.g. part geometry, on thermal stresses during and after production.

Withers and Bhadesia [11] explain why it is important to consider the sampling volume characteristics of stress measurement techniques and the types of stresses which may be of importance, before selecting one method over another. The Bridge Curvature Method can only be used to qualitatively assess macro-stresses (type I) which vary continuously over large distance. No residual stresses which vary over the grain scale (type II or inter-granular stresses) or atomic scale (type III) can be assessed.

According to Kandil et al. [12] numerous publications have been produced on the subject of residual stress and significant advances have been made recently to improve current measuring techniques. However, a number of important issues still remain including the uncertainties in the measurement, reliability and interpretation of results and, for many techniques, the general lack of standards. The current paper describes a way to assess the curling angle of a bridge structure with defined geometry, after removal by EDM from the base plate, as a measure for the residual stresses in the part. The curling angle is measured 10 times by a non-contact vision measuring system ( 5 times on each side of the part). From the measured data the mean value and standard deviation are calculated with a $95 \%$ confidence interval, quantifying the uncertainty in the measurement. As described above, the reliability of the Bridge Curvature Method is indicated by comparing the obtained results with results from literature. However, the obtained results can only be interpreted qualitatively.

\section{SUMMARY AND CONCLUSION}

The paper presents a new pragmatic method for stress assessment, namely the Bridge Curvature Method (BCM). The method uses a standardised technique to assess the angle of curvature $\alpha$ of SLM-made bridge structures cut off from the base plate as a measure for the thermal stresses. 
The uncertainties during measurement and the repeatability of the method are determined by

2 statistical analysis. The reliability of the method is indicated by comparing experimental results with findings from literature. Both literature review and performed experiments confirm that changes which reduce the high temperature gradient reduce the thermal stresses. Also optimising the orientation of the laser scan vectors can reduce thermal stresses or make their orientation less harmful.

The Bridge Curvature Method not only enables a new manner of assessing stresses, but also enables qualitatively comparing stress reducing strategies: i.e. different laser scan patterns, parameter settings and more fundamental process changes on residual stresses. One can e.g. conclude that the described heat treatment (which resulted in an $80 \%$ reduction of measured angle of curvature) reduces the thermal stresses more than optimising parameters for island scanning ( $36 \%$ reduction of measured angle).

\section{Acknowledgments}

The authors thank I.W.T. for the support through the SBO project DiRaMaP and the TUBITAK Research Council of Turkey for its financial support.

\section{References}

1. Mercelis P. Control of Selective Laser Sintering and Selective Laser Melting Processes. PhD thesis K.U.Leuven, Leuven, Belgium, April 2007.

2. Shiomi. M, Osakada K, Nakamura K, Yamashita T and Abe F. Residual stress within metallic model made by selective laser melting process. Annals of the CIRP 2004; 53/1: 195-198.

3. Mackwood A and Crafer R. Thermal modelling of laser welding and related processes: a literature review. Optics and Laser Technology 2005; 37: 99-115.

4. Cheng PJ and Lin SC. An analytical model for the temperature field in the laser forming of sheet metal. Journal of Materials Processing Technology 2000; 101: 260-267. 
5. Nickel AH, Barnett DM, Prinz FB. Thermal stresses and deposition patterns in layered manufacturing. Materials Science and Engineering 2001; A317/1: 59-64.

6. $\mathrm{Li} \mathrm{JF}$, Li L and Stott FH. Thermal stresses and their implication on cracking during laser melting of ceramic materials. Acta Materialia 2004; 52: 4385-4398.

7. Osakada $\mathrm{K}$ and Shiomi $\mathrm{M}$. Flexible manufacturing of metallic products by selective laser melting of powder. International Jounal of Machine Tools \& Manufacture 2006, 46: 11881193.

8. Over C, Meiners W, Wissenbach K, Hutfless $\mathrm{J}$ and Lindeman M. Rapid manufacturing of metal parts and tools using laser melting. Proceedings of WLT-Conference on Lasers in Manufacturing, Munich, Germany, June 24-26 2003; 265-269.

9. Niebling $\mathrm{F}$ and Otto A. FE-simulation of the selective laser sintering process of metallic powders. Proceedings of the LANE 2001, Erlangen, Germany, August 28-21 2001, 371382.

10. Bhadeshia HKDJ. Material factors. In: Totten GE, Howes MAH and Inoue T (eds), Handbook of Residual Stress and Deformation of Steel, USA: ASM International, 2002, pp. 3-10.

11. Withers PJ, Bhadesia HKDH. Residual stress part 1: Measurement techniques. Materials Science and Technology 2001; 17/4:355-365.

12. Kandil FA, Lord JD, Fry AT and Grant PV. A review of residual stress measurement methods - a guide to technical selection. Technical Report NPL Materials Centre, 2001.

13. Prime MB. Cross-sectional mapping of residual stresses by measuring the surface contour after cut. Journal of Engineering Materials and Technology 2001; 123: 162-168.

14. Wauthlé R. Verminderen van thermische spanningen bij selectief laser smelten. Master thesis K.U.Leuven (Dept. Mech. Eng.), Leuven, Belgium, 2009. 
1 15. Gilbert R and Shannon R. ASM Handbook Vol.4: Heat treating of titanium alloys, USA: ASM International, 1991, pp. 2043-2071.

3 16. Yasa E. Manufacturing by combining Selective Laser Melting and Selective Laser Erosion / Laser Re-melting. PhD Thesis, K.U.Leuven, Leuven, Belgium, January 2011. 